\title{
Efecto del factor de severidad y tiempo de almacenamiento sobre las características fisicoquímicas de Pasta Desamargada de Tarwi
}

\section{Effect of the severity factor and storage time on the physicochemical characteristics of Paste of Andean Lupine}

\author{
Rodolfo M. Vegas-Niño ${ }^{1 *}$ \& Zacarías Argomedo-Reyes ${ }^{1}$ \\ ${ }^{1}$ Universidad Nacional de Trujillo-Escuela de Ingeniería Agroindustrial-Filial Huamachuco. \\ *Autor corresponsal: Rodolfo M. Vegas-Niño,rvegas@unitru.edu.pe
}

\begin{abstract}
RESUMEN
El objetivo del presente trabajo de investigación fue evaluar el efecto del factor de severidad (considerado como la combinación de tiempo: $5,15,25 \mathrm{~min}$ y temperatura de autoclave: $121^{\circ} \mathrm{C}$ ) con el posterior tiempo de almacenamiento (hasta 32 días a $16^{\circ} \mathrm{C}$ y HR: $56 \%$ aproximadamente) sobre las características fisicoquímicas de pasta desamargada de tarwi (Lupinus mutabilis) variedad criolla. El método utilizado es el experimental. Entre las características fisicoquímicas evaluadas en la pasta de tarwi fueron contenido de sólidos, $\mathrm{pH}$, acidez titulable, sólidos solubles, índice el TBA, conductividad eléctrica y azúcares reductores. Un aumento de los factores de severidad (Log Ro) en el tratamiento térmico de pasta desamargada de tarwi provoca un descenso del $\mathrm{pH}$, un aumento de la acidez titulable, una mayor oxidación de las grasas, un aumento de la conductividad eléctrica, un aumento de los sólidos solubles y un aumento de azúcares reductores. Después del tratamiento térmico un mayor tiempo de almacenamiento a condiciones ambientales en frascos herméticos indujo un aumento de la acidez, una disminución del pH, un incremento en los índices de TBA, un aumento de la conductividad eléctrica; sin embargo, no ocasiona una variabilidad en los parámetros de sólidos solubles y de azúcares reductores.
\end{abstract}

Palabras claves: Pasta de Lupinus mutabilis, factor de severidad, características fisicoquímicas

ABSTRACT
The objective of the present research work was to evaluate the effect of the severity factor (considered as the combination of time: $5,15,25 \mathrm{~min}$ and autoclave temperature: $121^{\circ} \mathrm{C}$ ) with the subsequent storage time (up to 32 days at $16^{\circ} \mathrm{C}$ and $\mathrm{RH}: 56 \%$ approximately) on the physicochemical characteristics of debittered tarwi paste (Lupinus mutabilis) creole variety. The method used is the experimental. Among the physicochemical characteristics evaluated in the tarwi paste were solids content, $\mathrm{pH}$, titratable acidity, soluble solids, TBA index, electrical conductivity and reducing sugars. An increase in severity factors (Log Ro) in the thermal treatment of debittered tarwi pasta causes a decrease in $\mathrm{pH}$, an increase in titratable acidity, greater oxidation of fats, an increase in electrical conductivity, an increase in soluble solids and an increase in reducing sugars. After heat treatment, a longer storage time at ambient conditions in hermetic jars induced an increase in acidity, a decrease in $\mathrm{pH}$, an increase in TBA indices, an increase in electrical conductivity; however, it does not cause a variability in the parameters of soluble solids and reducing sugars

Key words: Paste of Lupinus mutabilis, factor of severity, characteristic chemical physical.

\section{INTRODUCCIÓN}

El tarwi es una leguminosa que se cultiva tradicionalmente en los Andes desde los 1,500 msnm (Navarrete, 2010), es rico en proteínas y grasas, con mayor frecuencia, su contenido proteico es superior al de la soya (Palacios et al., 2004).

La pasta de tarwi puede seguir diversas alternativas de procesamiento tales como: su conversión en "leche" (Baldeón, 2012), "yogurt" (Holguín et al., 2019), mermelada (Villarroel et al., 1996), torta (Tapia, 2015), salsas y espesante para jugos (Jacobsen \& Mujica, 2006).

Christensen (1989) hace referencia que la hidrólisis de biopolímeros (carbohidratos, lípidos y proteína) tiene como efecto una reducción del tamaño molecular, así como también cambios estructurales y de polaridad. Se incrementa el carácter hidrofílico por el aumento de la exposición de grupos carboxílicos libres y el carácter hidrófobo, por la exposición de unidades de aminoácidos apolares. La solubilidad de las proteínas parcialmente hidrolizadas mejora en todo el intervalo de $\mathrm{pH}$.

Overend Chornet (1987) desarrollaron el llamado factor ordinario de reacción o factor de severidad $\left(\mathrm{R}_{0}\right.$ o $\left.\log \mathrm{R}_{0}\right)$. La teoría está basada en el factor hidrólisis de biopolímeros, combinando los efectos de la temperatura, y el tiempo de reacción en un único parámetro. El factor de severidad es el logaritmo o el área bajo la curva del procesamiento térmico producto del remplazo de valores de temperatura empleando en cada unidad de tiempo durante todo el tratamiento térmico. Es de asumir que el tratamiento térmico afectará la composición de la pasta de tarwi promoviendo cambios en la composición química. Se define mediante la siguiente expresión (donde $\mathrm{T}_{\exp }$ es la temperatura en ${ }^{\circ} \mathrm{C}$ ):

$$
R_{0}=\int e x p^{\left(T_{\exp }-100 / 14.75\right)} \cdot d t
$$

El factor $\mathrm{R}_{0}$ se emplea para interpretar el grado de solubilización de los biopolímeros de la pasta de tarwi, permitiendo comparar tratamientos que se realizan en diferentes condiciones de tiempo y temperatura.

El tarwi se cultiva en el Ande Liberteño (Perú) y tiene un potencial de industrialización como pasta que permite combatir la desnutrición en los habitantes de la zona rural. Ante ello, la presente investigación tuvo como objetivo evaluar el efecto del factor de severidad y tiempo de almacenamiento sobre las propiedades fisicoquímicas de pasta desamargada de tarwi (Lupinus mutabilis) variedad criolla.

\section{MATERIALES Y MÉTODOS}

\section{Materia prima}

Se utilizó semillas de tarwi (Lupinus mutabilis) variedad criolla proveniente del distrito de Huamachuco, Provincia de Sánchez Carrión, Departamento de La Libertad. 
Tabla 1. Propiedades fisicoquímicas de la pasta desamargada de tarwi en función al tiempo de almacenamiento a condiciones de ambientales $\left(16^{\circ} \mathrm{C}\right.$ y HR: $\left.56 \%\right)$.

\begin{tabular}{lccccc}
\hline VARIABLES & \multicolumn{5}{c}{ Tiempo de almacenamiento (días) } \\
\cline { 2 - 6 } & $\mathbf{0}$ & $\mathbf{2}$ & $\mathbf{4}$ & $\mathbf{6}$ & $\mathbf{8}$ \\
\hline \% Sólidos & $24,3 \pm 0,1$ & $24,3 \pm 0,2$ & $24,3 \pm 0,1$ & $24,3 \pm 0,1$ & $24,2 \pm 0,2$ \\
\hline $\mathrm{pH}$ & $5,2 \pm 0,01$ & $5,02 \pm 0,02$ & $4,98 \pm 0,02$ & $4,91 \pm 0,03$ & $4,82 \pm 0,01$ \\
\hline $\begin{array}{l}\text { \% Acidez } \\
\text { (como ácido oleico) }\end{array}$ & $1,11 \pm 0,2$ & $1,68 \pm 0,1$ & $2,29 \pm 0,2$ & $2,94 \pm 0,3$ & $3,55 \pm 0,1$ \\
\hline $\begin{array}{l}\text { Conductividad eléctrica } \\
(\mu \text { s/cm) }\end{array}$ & $91 \pm 4$ & $217 \pm 2$ & $246 \pm 1$ & $251 \pm 3$ & $294 \pm 5$ \\
\hline Sólidos solubles ( ${ }^{\circ}$ Brix) & 2 & 2 & 3 & 4 & 4 \\
\hline $\begin{array}{l}\text { Índice del TBA(mg de } \\
\text { malonaldehído/kg de } \\
\text { muestra) }\end{array}$ & $39,0 \pm 0,2$ & $39,0 \pm 0,1$ & $40,6 \pm 0,3$ & $43,6 \pm 0,1$ & $46,8 \pm 0,2$ \\
\hline$\%$ Azúcares reductores & $0,41 \pm 0,3$ & $0,44 \pm 0,1$ & $0,47 \pm 0,1$ & $0,51 \pm 0,2$ & $0,60 \pm 0,1$ \\
\hline
\end{tabular}

El proceso de deslupinizado (desamargado) se logró remojando los granos por $24 \mathrm{~h}$, una posterior cocción por 4 minutos (3 veces) y un lavado constante con agua de la red pública por 7 días.

\section{Proceso de obtención de pasta de tarwi desamargada}

Se seleccionó los granos de tarwi a través de inspección visual separando los que se encuentran en mal estado (picado, quebrado, manchado, con presencia de insectos). Se realizó un lavado manual con agua destilada para eliminar restos de tierra. Se sumergió los granos en una solución de hipoclorito de sodio a $3 \mathrm{ppm}$ por 20 minutos con el fin de disminuir la carga microbiana.

Los granos de tarwi se lavaron con agua destilada con el propósito de eliminar restos de hipoclorito presentes en su superficie. Posteriormente se realizó un triturado en una licuadora casera en una proporción grano/agua 1:1 (p/p) obteniendo una pasta homogénea con un contenido de sólidos totales superiores al $24 \%$.

La masa total de la pasta se dividió en dos condiciones:

Condición I: Pasta de tarwi sin tratamiento térmico conocida como muestra "testigo" o "blanco", la cual se almacenó en frascos estériles y cerrados a condiciones ambientales. Los mismos que se evaluaron sus propiedades fisicoquímicas a los $0,2,4,6,8$ días.

Condición II: Pasta de tarwi sometida a tratamiento térmico en su propio frasco a $121^{\circ} \mathrm{C}$ y tiempo de mantenimiento de autoclave de 5, 15 y $25 \mathrm{~min}$, la cual se almacenó a condiciones ambientales para evaluar el efecto del factor de severidad (combinación tiempo-temperatura de autoclave) más el tiempo de almacenamiento sobre las características fisicoquímicas. La cantidad de pasta de tarwi por frasco fue de 140 gramos aproximadamente.

Entre las variables dependientes evaluadas en la pasta de tarwi tenemos:

a) Determinación del contenido de sólidos (ISO 638, 2008)

b) Determinación del pH (AOAC, 1990)

c) Determinación de la acidez titulable (NTP 203.070, 1977)

d) Determinación de sólidos solubles (NMX-F-103, 1982)

e) Determinación del índice el TBA (Ácido 2-Tiobarbitúrico) (Witas, 1978)

f) Determinación de conductividad eléctrica (Persano et al., 2004).

g) Determinación de azúcares reductores (Método Lane Eylon, NMX-F-312, 1978)

\section{RESULTADOS Y DISCUSIONES}

En la Tabla 1 muestra las propiedades fisicoquímicas de la pasta desamargada de tarwi. La tendencia del $\mathrm{pH}$ con el tiempo de almacenamiento es de manera inversa; en tanto con la acidez y conductividad eléctrica de manera directa. El valor de $\mathrm{pH}$ inicial del grano de tarwi fue de 5.53, el mismo que se acidifica con el transcurrir el tiempo. Su valor inicial es cercano a lo reportado por Apunte y León (2012) que establecen valores en torno a $5.86 \pm 0.02$. Se ha establecido que los cereales y leguminosas no presentan valores de $\mathrm{pH}$ muy bajos, así lo demuestra la harina de avena (pH: 6.02), trigo (pH: 5.70), soja (pH: 6.40), judía y lenteja (pH:6.5) (Rachel, 2012).

La pasta desamargada de tarwi presenta en un inicio valores de sólidos solubles de $2{ }^{\circ}$ Brix. Parte de esta concentración podría venir del aporte del contenido de sacarosa y almidón soluble que contiene el grano (Schoeneberger et al., 1982); sin embargo, hay un ligero aumento al aumentar el tiempo de almacenamiento producto posiblemente de acción microbiana.

El porcentaje de acidez de la pasta de tarwi desamargada a condiciones ambientales $\left(15^{\circ} \mathrm{C}\right)$ aumenta durante los días de almacenamiento, pudiendo llegar hasta un 3,55\% al octavo día (expresado como ácido oleico). Es de considerar que la acidez de las sustancias grasas es muy variable. Generalmente las pastas frescas o recién preparadas no contienen ácidos grasos libres o si los contienen los tienen en muy pequeñas cantidades. $\mathrm{Al}$ envejecer, especialmente si no han estado protegidos de la acción del aire y la luz su acidez aumenta lentamente al principio y con cierta rapidez después (Badui, 2006). Se puede considerar que la presencia natural de la acidez libre en las grasas a condiciones ambientales puede ser el resultado de la hidrólisis o descomposición lipolítica de algunos triglicéridos deducidos mayoritariamente por acción microbiana (Badui, 2006).

Si bien la conductividad eléctrica en un alimento es una medida de la concentración de iones, sales minerales o analitos que contiene (Badui, 2006). Schoeneberger et al. (1982) establecen que el calcio se encuentra principalmente en la cáscara, mientras que el fósforo en el endocarpio. Hay que tener presente que la pasta generada en el trabajo experimental fue producto del triturado de su cáscara conjuntamente con el endocarpio.

En el caso de soluciones acuosas, el valor de la conductividad es directamente proporcional a la concentración de sólidos disueltos; por tanto, cuanto mayor sea dicha concentración, mayor será la conductividad. 
Tabla 2. Factores de severidad (Log Ro) y su representación en porcentaje (\%) en la intensidad del tratamiento térmico de la pasta desamargada de tarwi.

\begin{tabular}{|c|c|c|c|c|c|c|}
\hline \multirow[b]{2}{*}{ Tiempo (minutos) } & \multicolumn{3}{|c|}{$\begin{array}{l}\text { Expresado como factor de severidad Ro a } \\
121^{\circ} \mathrm{C}\end{array}$} & \multicolumn{3}{|c|}{$\begin{array}{c}\text { Expresado como porcentaje de } \\
\text { influencia, } \% \text { a } 121^{\circ} \mathrm{C}\end{array}$} \\
\hline & 5 & 15 & 25 & 5 & 15 & 25 \\
\hline \multicolumn{7}{|l|}{ Etapa } \\
\hline Calentamiento & 22,43 & 22,77 & 22,88 & 12,92 & 10,56 & 8,64 \\
\hline Isotermo & 18,95 & 60,75 & 105,75 & 10,91 & 28,17 & 39,94 \\
\hline Enfriamiento & 132,30 & 132,12 & 136,15 & 76,17 & 61,27 & 51,42 \\
\hline Total Ro & 174 & 216 & 265 & 100,00 & 100,00 & $\begin{array}{c}100,0 \\
0\end{array}$ \\
\hline Log Ro & 2,24 & 2,33 & 2,42 & & & \\
\hline
\end{tabular}

Tabla 3. Efecto del factor de severidad y tiempo de almacenamiento (a condiciones ambientales) sobre el pH, acidez, conductividad eléctrica e índice de TBA de la pasta desamargada de semillas de tarwi.

\begin{tabular}{|c|c|c|c|c|c|}
\hline $\begin{array}{l}\text { Factor de } \\
\text { severidad Log Ro }\end{array}$ & $\begin{array}{l}\text { Tiempo } \\
\text { (días) }\end{array}$ & pH & $\begin{array}{c}\text { \% Acidez } \\
\text { (como ác. oleico) }\end{array}$ & $\begin{array}{c}\text { Conductividad } \\
\text { eléctrica } \\
(\mu \mathrm{S} / \mathrm{cm})\end{array}$ & $\begin{array}{c}\text { Índice de TBA } \\
\text { (mg malonaldehído } \\
\text { por kg de pasta) }\end{array}$ \\
\hline \multirow{5}{*}{2,24} & 0 & 5,51 & 1,19 & 74,5 & 57,7 \\
\hline & 8 & 5,46 & 1,72 & 79,4 & 62,4 \\
\hline & 16 & 5,44 & 2,00 & 79,4 & 78,0 \\
\hline & 24 & 5,34 & 2,00 & 88,5 & 65,5 \\
\hline & 32 & 5,32 & 2,00 & 89,0 & 85,8 \\
\hline \multirow{5}{*}{2,33} & 0 & 5,45 & 1,13 & 108,6 & 85,8 \\
\hline & 8 & 5,56 & 1,71 & 111,4 & 85,8 \\
\hline & 16 & 5,40 & 2.00 & 113,1 & 106,1 \\
\hline & 24 & 5,37 & 1.99 & 118,2 & 95,2 \\
\hline & 32 & 5,29 & 1.99 & 123,4 & 85,8 \\
\hline \multirow{5}{*}{2,42} & 0 & 5,42 & 1,10 & 121,7 & 142,0 \\
\hline & 8 & 5,52 & 1,62 & 150,7 & 142,0 \\
\hline & 16 & 5,44 & 1.72 & 158,3 & 182,5 \\
\hline & 24 & 5,41 & 1.78 & 164,2 & 215,3 \\
\hline & 32 & 5,39 & 1,64 & 168.8 & 103,0 \\
\hline
\end{tabular}

En el almacenamiento de la pasta desamargada de tarwi se observó un incremento de su conductividad eléctrica a condiciones ambientales $\left(16^{\circ} \mathrm{C}\right)$, debido mayoritariamente a la proliferación de microorganismos más que a la hidrólisis de polímeros como almidón o cadena de ácidos grasos. Se constató que a partir del cuarto día de almacenamiento a condiciones ambientales la pasta de tarwi emanó olores fétidos debido a la acción microbiana. A dicho tiempo se obtuvo 30 x10^6 UFC de hongos mesófilos por gramo de muestra.

Se ha establecido que severidades (Log Ro) de 3.65-4.81 influye en la obtención de pentosas de materiales lignocelulósicos a partir de maderas duras y que temperaturas superiores a $200^{\circ} \mathrm{C}$ pueden solubilizar parcialmente la celulosa (Kim et al., 2014).

Con el propósito de darle mayor estabilidad a la pasta desamargada de tarwi ( $24 \%$ de sólidos), se realizó un proceso de tratamiento térmico a vapor a $121^{\circ} \mathrm{C}$ (en autoclave) y tiempos de 5, 15 y 25 min generando factores de severidad (Log Ro) de 2.24, 2.33 y 2.42 respectivamente. En ella se observó que al aumentar el tiempo de tratamiento térmico se genera un pequeño descenso del valor de $\mathrm{pH}$ y un ligero aumento del porcentaje de acidez expresado como ácido oléico. En la Tabla 3 se presenta el efecto del factor de severidad y tiempo de almacenamiento sobre el $\mathrm{pH}$, acidez, conductividad eléctrica e índice de TBA de la pasta desamargada de semillas de tarwi.
El pH inicial de la pasta de tarwi fue de 5,23 $\pm 0,01$, en tanto; después de someterlo a tratamiento térmico a severidad de 2.24 fue de 5.51; a severidad de 2.33 de 5.45 y a severidad de 2.42 de 5.42. En todos ellos el efecto predominante es el ligero aumento del valor de $\mathrm{pH}$ con referencia al valor inicial. Chau et al. (1997) consideran que los valores de $\mathrm{pH}$ de las legumbres producen un incremento de dicho valor cuando estas son procesadas térmicamente, debido probablemente a la solubilización de aminoácidos básicos. Por otro lado, el ligero descenso del valor de $\mathrm{pH}$ y consecuente aumento de la acidez de la pasta de tarwi con el incremento del tiempo de almacenamiento se deba mayoritariamente a la acción microbiana que origina una ligera acidificación del medio.

Autores como Lawford y Rousseau (1993) consideran que un aumento del factor de severidad en medios muy acuosos genera un descenso del valor de $\mathrm{pH}$ del medio debido al desprendimiento de grupos acetilos que se encuentran como sustituyentes (grupos adheridos) en la cadena de hemicelulosa perteneciente a la cáscara del tarwi. Se establece que en procesos de tratamiento térmico las uniones éter heterocíclicas de las hemicelulosas son las más susceptibles a este tipo de reacción, generando oligosacáridos de distinto grado de polimerización y liberando los grupos acetilo unidos a la cadena principal. En etapas de reacción más severas, los iones hidronio generados de la autoionización de ácido acético 
también actúan como catalizadores en la degradación de los polisacáridos. En las condiciones de operación normalmente empleadas en los tratamientos hidrotérmicos $\left(200-250^{\circ} \mathrm{C}\right)$, la formación de iones hidronio provenientes del ácido acético es más importante que la autoionización del agua (Heitz et al., 1986), cuyo efecto está limitado a las fases iniciales de reacción. Adicionalmente, los ácidos urónicos (constituyente de los materiales lignocelulósicos) pueden también contribuir a la generación de iones hidronio, pero sus efectos no están bien establecidos (Conner, 1984).

El índice de TBA es un parámetro que mide el grado de oxidación de las grasas. Grasas oxidadas presentan valores de TBA cada vez mayores y de conductividades eléctricas mayores (Badui, 2006). Por otro lado, Tinsky et al. (1995) establecen que debido a la multiplicación microbiana se produce un cambio en la composición iónica de los alimentos originando un aumento en los valores de conductividad eléctrica.

Una tendencia en los procesos del tratamiento térmico llevados en el laboratorio es que a mayor factor de severidad mayor son los valores de conductividad térmica. Esta correlación directa la explican Persano y Piro (2004) al considerar que la intensidad del tratamiento térmico origina hidrólisis de las cadenas de biopolímeros produciendo electrolitos, sales minerales disueltas, ácidos orgánicos y aminoácidos.

La autoxidación es el deterioro más común de las grasas y aceites y se refiere a la oxidación de los ácidos grasos insaturados, pero también se presenta con otros compuestos de interés biológico, como la vitamina A y los carotenoides (Badui, 2006). Tratamientos más intensos llevan consigo mayores valores de conductividad eléctrica. La tendencia mayoritariamente generalizada es que a medida que aumenta el tiempo de almacenamiento el índice de TBA también aumenta.

En oleaginosas con contenidos altos en grasa, los tratamientos térmicos con aplicación de presión pueden producir una parcial y pequeña isomerización de ácidos grasos "cis" a "tras" y una desactivación de lipasas y peroxidasas que confieren una mayor estabilidad a los productos evitando su enranciamiento. Sin embargo, se ha establecido que a condiciones de sobrecalentamiento pueden formar compuestos de oxidación como peróxidos, dímeros y polímeros y ácidos grasos (Apelt, 1987)

Saavedra y Vásquez (2013) consideran que la rancidez oxidativa no tiene mayor influencia en el deterioro de la harina no desgrasada de tarwi por tanto es necesario un mejor control de la rancidez hidrolítica. En pruebas experimentales determinaron que a medida que transcurre el tiempo de almacenamiento existe un aumento de los ácidos grasos libres expresados como \% de ácido oleico. A condiciones de humedad relativa del $65 \%$ y temperatura de $25^{\circ} \mathrm{C}$ la harina de tarwi completa incrementa el porcentaje de ácidos grasos libres de 1 a $2.5 \%$ en los primeros 15 días y $5.5 \%$ en los primeros 45 días.

Se debe tomar en consideración que dentro de la composición de ácidos grasos en el grano de tarwi existe mayor presencia de ácido linoleico $(49,68 \%)$, oleico $(30,91 \%)$ y palmítico $(9,73 \%)$ (Ramos, 2006). Se considera que la oxidación de los lípidos insaturados en los alimentos produce compuestos aldehídicos volátiles que, junto con los alcoholes derivados de su reducción, son los responsables de los olores desagradables que desarrollan los alimentos durante su procesado o almacenaje, siendo la lipoxigenasa la responsable principal del proceso y que se inactiva por acción de tratamiento térmico (Zamora et al. 1991).

El ligero aumento de sólidos solubles en la pasta desamargada de tarwi antes del tratamiento térmico $\left(2^{\circ}\right.$ Brix $)$ y después de este para un factor de severidad de 2.42 fue de $4^{\circ}$ Brix (Tabla 4), esto se debería probablemente a la hidrólisis parcial de la fracción amorfa del almidón (la más fácil de hidrolizar) más que a la fracción lignocelulósica. Taherzadeh et al. (1997) consideran que la hidrólisis de los materiales lignocelulósicos es un proceso muy complejo. Entre los factores que influyen en la cinética del proceso se encuentran tamaño de partícula, relación sólido-líquido, temperatura y tiempo de reacción. Por otra parte, Kosaric et al. (1983) consideran además la configuración y grado de polimerización de las macromoléculas y su interacción con proteínas y elementos minerales factores que influyen en el proceso hidrolítico.

Tabla 4: Efecto del factor de severidad y tiempo de almacenamiento sobre los sólidos solubles y azúcares reductores en pasta desamargada de semillas de tarwi.

\begin{tabular}{|c|c|c|c|}
\hline $\begin{array}{c}\text { Log } \\
\text { Ro }\end{array}$ & $\begin{array}{c}\text { Tiempo } \\
\text { (días) a } \\
\text { condiciones } \\
\text { ambientales } \\
\end{array}$ & $\begin{array}{c}\text { Sólidos } \\
\text { Solubles } \\
\left({ }^{\circ} \text { Brix) }\right.\end{array}$ & $\begin{array}{c}\% \\
\text { Azúcares } \\
\text { reductores }\end{array}$ \\
\hline \multirow{5}{*}{2,24} & 0 & 3 & 0,86 \\
\hline & 8 & 4 & 0,86 \\
\hline & 16 & 4 & 0,86 \\
\hline & 24 & 4 & 1,05 \\
\hline & 32 & 4 & 1,05 \\
\hline \multirow{5}{*}{2,33} & 0 & 3 & 0,94 \\
\hline & 8 & 4 & 0,94 \\
\hline & 16 & 5 & 0,93 \\
\hline & 24 & 5 & 0,96 \\
\hline & 32 & 5 & 1,00 \\
\hline \multirow{5}{*}{2,42} & 0 & 4 & 1,04 \\
\hline & 8 & 5 & 1,04 \\
\hline & 16 & 6 & 0,97 \\
\hline & 24 & 6 & 1,22 \\
\hline & 32 & 6 & 1,24 \\
\hline
\end{tabular}

El ligero aumento de azúcares reductores (de 0,41 a 1,04\% expresado como glucosa equivalente) de la pasta desamargada de tarwi después del tratamiento térmico de severidad 2.42 se debería probablemente a la glucosa generada de la hidrólisis parcial del almidón. Se ha concebido que, a tratamientos moderados de hidrólisis, la glucosa generada de fuentes vegetales proviene del almidón, especialmente de la región amorfa (Sánchez et al., 2004), mientras que a tratamientos más severos la glucosa mayoritariamente proviene de la región cristalina del almidón o de la fracción celulósica (Taherzadeh et al., 1997). Los azúcares libres contenidos en la harina de lupino son mayoritariamente representados por glucosa y galactosa, cada uno a una concentración en el intervalo de 30 a $40 \mathrm{~g} / \mathrm{kg} \mathrm{ms}$ (Glencross, 2004).

Después del tratamiento térmico, la pasta de tarwi desamargada presenta un ligero oscurecimiento al aumentar la intensidad de tratamiento térmico. Esto se debería a que tiene lugar la reacción de Maillard, producida por condensación química entre aminoácidos y azúcares reductores (Dendy, 
2001). Entre los productos de la reacción de Maillard se incluyen compuestos volátiles de bajo peso molecular como alcoholes, cetonas, aldehídos, éteres, ésteres y componentes heterocíclicos como el furfural (Khan et al., 1995). También se forman compuestos menos volátiles con peso molecular medioalto, tales como polifenoles, polipéptidos y pigmentos (Mastrocola et al., 2000). Se ha establecido que los furanos, productos predominantes de la reacción de Maillard, son los responsables del color caramelo (Shibamoto, 1989).

Algunas investigaciones como la de Ashoor y Zent (1984) consideran el efecto del pH en la reacción de Maillard. Se ha demostrado que en general la reacción es inhibida por valores de $\mathrm{pH}$ bajos y favorecida por valores altos. Asimismo, la velocidad de reacción es máxima cuando la actividad de agua se encuentra en el intervalo de 0,6- 0,7 (Warmbier et al., 1976). La pasta de tarwi presentó un valor de $\mathrm{pH}$ inicial de 5.23 y un contenido de agua del $76 \%$ lo cual favorece el empardeamiento.

Para evaluar si el factor de severidad por tratamiento térmico y el tiempo de almacenamiento a condiciones ambientales ejercen una influencia significativa sobre las características fisicoquímicas $(\mathrm{pH}$, acidez, sólidos solubles, índice del TBA, conductividad eléctrica, azúcares reductores) de la pasta de tarwi se realizó un análisis de varianza (ANOVA). En ella se determinó un valor de p $<0.0001$, el cual es menor al valor estándar de 0.05 estableciéndose efecto significativo sobre las variables antes mencionadas.

\section{CONCLUSIONES}

Un aumento de los factores de severidad (Log Ro) en el tratamiento térmico de pasta desamargada de tarwi provoca un descenso de los valores de $\mathrm{pH}$ y un aumento de la acidez titulable expresada como ácido oleico, una mayor oxidación de las grasas reflejándose en un incremento de índice TBA (expresado como $\mathrm{mg}$ de malonaldehído/kg muestra) así como un aumento de la conductividad eléctrica producto de la hidrólisis de las cadenas de biopolímeros (almidón, proteína y lípidos principalmente).

Un aumento de los factores de severidad (Log Ro) en el tratamiento térmico de pasta desamargada de tarwi provoca un aumento de los sólidos solubles (expresado como ${ }^{\circ} \mathrm{Brix}$ ) y de azúcares reductores (expresado como glucosa equivalente). Una vez generada estás estructuras el tiempo de almacenamiento de la pasta a condiciones ambientales hasta 32 días no ocasiona una variabilidad en los parámetros antes mencionados.

\section{REFERENCIAS BIBLIOGRÁFICAS}

AOAC. (1990). Official methods of analysis of the Association of Official Analytical Chemists, $15^{\text {a }}$ ed. Ed. Helrich, K.; Arlington, VA. USA ALINORM 06/29/26. 2006. New York. APELT, J. (1987). En 6th European Symposium on Poultry Nutrition. Konigslutter, Germany. Citado por Gorrachategui M (2010) Efecto del tratamiento de las materias primas sobre su valor nutricional. XXVI Curso de Especialización FEDNA. 4 y 5 de noviembre p51-112.

Apunte, G. y León, G. (2012). Utilización de harina de chocho (Lupinus mutabilis) como ingrediente en la elaboración de pan [Tesis de pregrado, Escuela Superior Politécnica del Litoral, Guayaquil-Ecuador]. https://www.dspace.espol.edu.ec/bitstream/123456789/2455 3/1/Utilizacion $\% 20 \mathrm{de} \% 20$ harina $\% 20 \mathrm{de} \% 20$ chocho $\% 20$ en $\%$ 20la\%20elaboracion\%20de\%20pan.pdf
Ashoor, S. \& Zent, J. (1984). Maillard browning of common amino acids and sugars. Journal Food Science, 49, 12061207. https://doi.org/10.1111/j.1365-2621.1984.tb10432.x

Badui, S. (2006). Química de los Alimentos. 4a ed. Estado de México: Pearson Educación de México, S.A.

Baldeón, P. (2012). Procesamiento del chocho (Lupinus mutabilis sweet) para la obtención de leche y yogurt como alimentos alternativos de consumo humano [Tesis Universidad de Guayaquil-Ecuador]. http://repositorio.ug.edu.ec/bitstream/redug/1928/1/TESIS_ CHOCHO_PDF.pdf

Chau, C., Cheung, P. \& Wong, Y. (1997). Functional properties of protein concentrates from three Chinese indigenous legume seeds. Journal of Agricultural and Food Chemistry, 45,7, 2500-2503. https://doi.org/10.1021/jf970047c

Conner, A. (1984). Kinetic modeling of hardwood prehydrolysis. Part I: Xylan removal by water prehydrolysis. Wood Fiber Science, 16, 268-277. https://core.ac.uk/download/pdf/236632104.pdf

Dendy, D., Bogdan, J. \& Dobraszczyk, A. (2001). Cereales y productos derivados: química y tecnología. 1a ed. Zaragoza Editorial Acribia.

Christensen, F. (1989). Enzyme technology versus engineering technology in the food industry. Biotechnology and Applied Biochemistry, 11, 249-265.

Glencross, B. (2004). Lupins. Aqua Feeds: Formulation \& Beyond, Research Division, Department of Fisheries, North Beach, Australia. Volume 1(2): 15 pp.

Lawford, H. y Rousseau, J. (1993). Effects of pH and acetic acid on glucose and xylose metabolism by a genetically engineered ethanologenic Escherichia coli. Applied Biochemistry and Biotechnology, (39), 301-322. DOI: 10.1007/BF02918999

Mastrocola, D., Munari, M., Cioroi, M. \& Lerici, C. (2000). Interaction between Maillard reaction products and lipid oxidation in starch-based model systems. Journal of the Science of Food and Agriculture, (80), 684-690. DOI: 10.1002/(SICI)1097-0010(20000501)80:6<684:AIDJSFA589>3.0.CO;2-3.

Navarrete, V. (2010). Extracción, refinación, y caracterización físico-química y nutracéutica del aceite de chocho (Lupinus mutabilis sweet) [Tesis de pregrado, Escuela Superior Politécnica de Chimborazo, Ecuador]. http://dspace.espoch.edu.ec/bitstream/123456789/731/1/56T 00249.pdf

NMX-F-312. (1978). Normas Mexicanas. Dirección General de Normas. Determinación de azúcares reductores en alimentos.

NMX-F-103. (1982). Normas Mexicanas. Dirección General de Normas. Determinación de Grados Brix.

NTP 203.070. (1977). Norma Técnica Peruana. Productos elaborados a partir de frutas y otros vegetales. Determinación de acidez.

Overend, R. \& Chornet, E. (1987). Fractionation of lignocellulosics by steam- aqueous pretreatments [and discussion]. Philosophical Transactions of the Royal Society A: Mathematical, physical and engineering sciences: 321, 523-536. doi: 10.1098/rsta.1987.0029

Palacios, A., Demetrio, M. \& Espinoza, L. (2004). Obtención del alcohol a partir de la malta de Lupinus mutabilis (tarwi) [Tesis de Pregrado, Universidad Nacional del Centro del Perú] http://www.sidalc.net/cgibin/wxis.exe/?IsisScript=CIDAB .xis\&method $=$ post $\&$ format $\mathrm{o}=2$ \& cantidad $=1$ \&expresion $=\mathrm{mfn}=012003$

Persano, L., Piro, R., Runeau, É. Guyot-Declerck, C. \& Ivanov, T. (2004). Main European unifloral honeys: descriptive sheets. Apidologie, 35 (1), $\quad$ S38-S81. 
https://www.apidologie.org/articles/apido/pdf/2004/06/MH S06.pdf

Rachel, T. (2012). Impacto del procesamiento sobre la pared celular y las propiedades hipoglucémicas y tecnofuncionales de leguminosas [Tesis Doctoral, Universidad Autónoma de Madrid].

https://digital.csic.es/bitstream/10261/101597/1/tecnofunciona les\%20de\%20leguminosas.pdf

Ramos, E. (2006).Utilización de diversas leguminosas grano en la producción de le leche de cabra. Análisis de su valor nutritivo y calidad de la leche producida [Tesis Doctoral, Universidad de Granada]. https://digibug.ugr.es/bitstream/handle/10481/1366/164977 9x.pdf?sequence $=1 \&$ isAllowed $=y$

Saavedra, D. \& Vásquez, M. (2013). Efecto de la temperatura, tiempo y tamaño de partícula en la aplicación de antioxidantes del almacenamiento de harina de tarwi no desgrasada [Tesis de pre grado, Universidad Nacional de Trujillo]. https://dspace.unitru.edu.pe/handle/UNITRU/3547

Sánchez, G., Pilcher, L., Roslander, C., Modig, T., Galbe, M. \& Lidén, G. (2004). Dilute-acid hydrolysis for fermentation of the bolivian straw material paja brava. Bioresource Technology, https://doi.org/10.1016/j.biortech.2003.11.003

Shibamoto, T. (1989). Thermal generation of aromas. Volatile flavor chemicals formed by the Maillard reaction. ACS Symposium Series 409, Washington, DC, USA, p. 134-142.

Taherzadeh, M., Niklasson, C. \& Lidén, G. (1997). Acetic acid- P friend or foe in anaerobic batch conversion of glucose to ethanol by Saccharomyces cerevisiae. Chemical Engineering Science, 52(15),2653-2659. https://doi.org/10.1016/S00092509(97)00080-8

Tapia, M. (2015). El tarwi, lupino andino tarwi, tauri o chocho (Lupinus mutabilis Sweet) Proyecto: "Mujeres Andinas en Camino: Promoción del producto tarwi de la provincia de Huaylas hacia el mercado nacional e internacional en el marco rural del desarrollo sostenible" CF 019-2014-FIP. http://fadvamerica.org/wp-

content/uploads/2017/04/TARWI-espanol.pdf
Tinsky, M., Zoguri, S. \& Pebs, E. (1995). Early detection of clinical and suclinical mastitis using an on line lectrica conductivity devue on the perlor. Proceeding of th 3er internacional mastitis seminar.www.veterinaria.org/revistas/redvet/n030305/0305 16.pdf.

Witas, A. (1978). Determination of oxidation degree of fish oils by thiobarbituric acid method with alkaline hydrolysis. Die Nahrung, $\quad 22 \quad$ (2): 139-147. https://doi.org/10.1002/food.19780220203

Villarroel, M., Biolley, E., Larenas, G., Witting, E. \& Diaz, V. (1996). Caracterización química, sensorial y clínica de mermelada de lupino. Archivos Latinoamericanos de Nutrición, $46 \quad$ (3):234-241. https://www.alanrevista.org/ediciones/1996/3/art-10/

Warmbier, H., Schnickels, R. \& Labuza, T. (1976). Effect of glycerol on non enzymatic browning in a solid intermediate moisture food system. Journal of Food Science, 41, 521- 531. https://doi.org/10.1111/j.1365-2621.1976.tb00663.x

Zamora, R., Hidalgo, F. \& Alalz, M. (1991). Alteraciones bioquímicas de los lípidos en los alimentos vegetales I. Formación de los hidroperóxidos lipídicos. Instituto de la Grasa y sus Derivados, 42 (2),155-162. DOI:10.3989/gya.1991.v42.i2.1265.
Presentado: 08/08/2021 Aceptado: 28/08/2021 Publicado: 31/01/2022 